Circular photogalvanic effect in $\mathrm{HgTe} / \mathrm{CdHgTe}$ quantum well structures

This article has been downloaded from IOPscience. Please scroll down to see the full text article.

2010 Semicond. Sci. Technol. 25095005

(http://iopscience.iop.org/0268-1242/25/9/095005)

View the table of contents for this issue, or go to the journal homepage for more

Download details:

IP Address: 128.111.14.21

The article was downloaded on 11/08/2010 at 02:29

Please note that terms and conditions apply. 


\title{
Circular photogalvanic effect in HgTe/CdHgTe quantum well structures
}

\author{
B Wittmann ${ }^{1}$, S N Danilov ${ }^{1}$, V V Bel'kov ${ }^{2}$, S A Tarasenko ${ }^{2}$, E G Novik ${ }^{3}$, \\ H Buhmann ${ }^{3}$, C Brüne ${ }^{3}$, L W Molenkamp ${ }^{3}$, Z D Kvon ${ }^{4}$, N N Mikhailov', \\ S A Dvoretsky ${ }^{4}$, N Q Vinh ${ }^{5,7}$, A F G van der Meer ${ }^{5}$, B Murdin ${ }^{6}$ and \\ S D Ganichev ${ }^{1}$ \\ ${ }^{1}$ Terahertz Center, University of Regensburg, 93040 Regensburg, Germany \\ ${ }^{2}$ Ioffe Physical-Technical Institute of the Russian Academy of Sciences, 194021 St Petersburg, Russia \\ ${ }^{3}$ Physical Institute (EP3), University of Würzburg, 97074 Würzburg, Germany \\ ${ }^{4}$ Institute of Semiconductor Physics, 630900 Novosibirsk, Russia \\ ${ }^{5}$ FOM Institute for Plasma Physics ‘Rijnhuizen', PO Box 1207, NL-3430 BE Nieuwegein, \\ The Netherlands \\ ${ }^{6}$ University of Surrey, Guildford, GU2 7XH, UK \\ E-mail: sergey.ganichev@physik.uni-regensburg.de
}

Received 14 June 2010, in final form 14 June 2010

Published 5 August 2010

Online at stacks.iop.org/SST/25/095005

\begin{abstract}
We describe the observation of the circular and linear photogalvanic effects in $\mathrm{HgTe} / \mathrm{CdHgTe}$ quantum wells. The interband absorption of mid-infrared radiation as well as the intrasubband absorption of terahertz radiation in the quantun well structures is shown to cause a $d c$ electric current due to these effects. The photocurrent magnitude and direction varies with the radiation polarization state and crystallographic orientation of the substrate in a simple way that can be understood from a phenomenological theory. The observed dependences of the photocurrent on the radiation wavelength and temperature are discussed in terms of a microscopic model.
\end{abstract}

(Some figures in this article are in colour only in the electronic version)

\section{Introduction}

High mobility HgTe quantum well (QW) structures are attracting rapidly growing attention due to the peculiar band structure properties. HgTe QWs exhibit an inverted band structure ordering for QWs exceeding a certain critical width, i.e. the ordering of electron- and hole-like states is interchanged [1,2]. In this kind of QW the structural inversion asymmetry leads to a strong Rashba spin-orbit splitting with energies of the order of $30 \mathrm{meV}$ [3]. Furthermore, the quantum spin Hall effect [2] has been observed and manifests itself in the formation of two spin polarized counter propagating one-dimensional helical edge channels which results in a quantized conductance without magnetic field. The inverted band structure and strong spin-orbit interaction also give rise to unusual opto-electronic phenomena, e.g. a recently observed nonlinear magneto-gyrotropic photogalvanic effect

7 Present address. ITST, Department of Physics, University of California, Santa Barbara, CA 93106-4170, USA.
[4]. Photogalvanic effects (PGE), in particular the circular photogalvanic effect (CPGE), in low-dimensional structures have been proved powerful for the study of non-equilibrium processes in semiconductor QWs yielding information on their point-group symmetry, inversion asymmetry degree of QWs, details of the band spin-splitting, and on processes of electron momentum, spin, and energy relaxation (for a recent review see [5]). The CPGE is a photon-helicity-dependent photocurrent, caused by a transformation of the photon angular momenta into a translational motion of charge carriers. Microscopically, the conversion can be due to spin-dependent mechanisms [6-8] or orbital effects $[9,10]$. The mechanisms may, as shown theoretically $[11,12]$, contain contributions associated with the Berry curvature and side jumps giving rise to a macroscopic $d c$ current quadratic in the amplitude of the $a c$ electric field.

Here we report the observation and investigation of the CPGE in HgTe QW structures with different growth direction induced by terahertz (THz) as well as mid-infrared radiation (MIR). Our investigations show that the CPGE can be 
effectively generated in $\mathrm{HgTe}$ QWs with a strength of about an order of magnitude larger than that observed in GaAs, InAs and SiGe low-dimensional structures [5]. This large photocurrent magnitude is of particular importance for an optimization of all-electric semiconductor room temperature detectors, which are based on PGE and provide information about the polarization state of the laser radiation [13]. We present the phenomenological theory of the linear photogalvanic effect (LPGE) and CPGE in two-dimensional channels with pointgroup symmetry corresponding to $\left(\begin{array}{llll}0 & 0 & 1\end{array}\right)$ - and ( $\left.\begin{array}{llll}0 & 1 & 3\end{array}\right)$-oriented structures and compare the results with experimental data on polarization dependences. We show the results of the band structure calculations and discuss the possible mechanisms of infrared/terahertz radiation absorption.

\section{Experimental details}

The experiments were carried out on $n$-type $\mathrm{Hg}_{0.3} \mathrm{Cd}_{0.7} \mathrm{Te} / \mathrm{HgTe} / \mathrm{Hg}_{0.3} \mathrm{Cd}_{0.7} \mathrm{Te} \quad \mathrm{QW}$ structures of the (0 0101$)$ and (0 1 13$)$ crystallographic orientations and various QW widths $L_{W}$. Structures with the $\left(\begin{array}{lll}0 & 0 & 1\end{array}\right)$ surface orientation were grown by molecular beam epitaxy (MBE) on $\mathrm{Cd}_{0.96} \mathrm{Zn}_{0.04}$ Te substrates and have $L_{W}$ either $8 \mathrm{~nm}$ or $22 \mathrm{~nm}$. Structures with the $\left(\begin{array}{lll}0 & 1 & 3\end{array}\right)$ surface orientation were grown on GaAs substrates by a modified MBE method [14] and have $L_{W}=21 \mathrm{~nm}$. Samples with the sheet density of electrons $n_{s}$ from $1 \times 10^{11} \mathrm{~cm}^{-2}$ to $2 \times 10^{12} \mathrm{~cm}^{-2}$, and the mobility at $4.2 \mathrm{~K}$ in the range between $5 \times 10^{4}$ and $5 \times 10^{5} \mathrm{~cm}^{2} / \mathrm{Vs}$ were studied in the temperature range from $4.2 \mathrm{~K}$ up to room temperature. While for $\left(\begin{array}{lll}0 & 0 & 1\end{array}\right)$ QWs all edges were obtained by cleaving, for (0 13) samples two edges were prepared by cleaving and the others were cut normal to them. We fabricated two pairs of ohmic contacts prepared by thermal in-bonding and centred in the middle of sample edges. In this way we

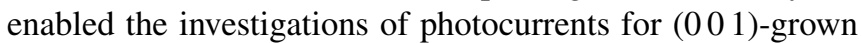
QWs along the $x \|\left[\begin{array}{lll}1 & 1 & 0\end{array}\right]$ and $y \|\left[\begin{array}{lll}1 & 1 & 0\end{array}\right]$ crystallographic directions and in (0 13)-oriented samples along the $x^{\prime}$ and $y^{\prime}$ directions. The photocurrent was measured in unbiased structures via the voltage drop across a $50 \Omega$ load resistor. The voltage was recorded with a storage oscilloscope. The samples were mounted in an optical cryostat with $z$-cut crystal quartz windows which allowed us to study photocurrents in the temperature range from $4.2 \mathrm{~K}$ up to room temperature.

The photocurrents were induced by applying mid-infrared or terahertz laser radiation. As a radiation source in the MIR range between $6 \mu \mathrm{m}$ and $15 \mu \mathrm{m}$ (corresponding to photon energies from $\hbar \omega=206.7 \mathrm{meV}$ to $82.7 \mathrm{meV}$ ) we used a frequency-tunable free electron laser 'FELIX' at FOMRijnhuizen in the Netherlands [15]. The output pulses of light from FELIX were chosen to be $\approx 6$ ps long, separated by $40 \mathrm{~ns}$, in a train (or 'macropulse') of $7 \mu$ s duration. The macropulses had a repetition rate of $5 \mathrm{~Hz}$. The extraction of the absolute photocurrent magnitude in response to such short pulses is not an easy task. To calibrate the data we used $\approx 100 \mathrm{~ns}$ long pulses of a transversely excited atmospheric pressure (TEA) $\mathrm{CO}_{2}$ laser with a fixed operating wavelength of $\lambda=9.6 \mu \mathrm{m}$ (corresponding photon energy $\hbar \omega=129 \mathrm{meV}$ ) and power $P \simeq 2 \mathrm{~kW}$. The wavelength dependence of the current from
FELIX was scaled to coincide with the $\mathrm{CO}_{2}$ laser result. For the measurements in the terahertz range we used a molecular gas laser, which was pumped optically by the TEA $\mathrm{CO}_{2}$ laser [16]. With $\mathrm{NH}_{3}$ as the active laser gas, we obtain $100 \mathrm{~ns}$ pulses of linearly polarized radiation at wavelengths $\lambda=90,148$ and $280 \mu \mathrm{m}$ (corresponding photon energies $\hbar \omega$ are 13.7, 8.4 and $4.4 \mathrm{meV}$ ). We also used $\mathrm{D}_{2} \mathrm{O}$ and $\mathrm{CH}_{3} \mathrm{~F}$ as laser gases to obtain radiation with $\lambda=385$ and $496 \mu \mathrm{m}(\hbar \omega=3.2$ and $2.5 \mathrm{meV})$, respectively. The peak power of $\mathrm{THz}$ radiation used in our experiments was in the range from $3 \mathrm{~kW}$ to $30 \mathrm{~kW}$. Radiation was applied with an angle of incidence $\theta_{0}$ varying from $-30^{\circ}$ to $+30^{\circ}$ to the QW normal in the $(x z)$ plane, see the inset of figure 2. To investigate the CPGE we use elliptically polarized light. The polarization of the laser beam was modified from linear to elliptical (and circular) by means of ZnSe Fresnel rhombus for the mid-infrared radiation and crystal quartz $\lambda / 4$-plates for the $\mathrm{THz}$ radiation. The radiation helicity $P_{\text {circ }}$ of the incident light varies from $-1\left(\sigma_{-}\right)$to $+1\left(\sigma_{+}\right)$according to $P_{\text {circ }}=\sin 2 \varphi$, where $\varphi$ is the angle between the initial plane of the laser radiation polarization and the optical axis of the quarter-wave polarizer. The light polarization states for some characteristic angles $\varphi$ are sketched on the top of figure 2 .

In our samples, MIR and $\mathrm{THz}$ radiations induced photocurrents due to direct band-to-band optical transitions or indirect intrasubband (Drude-like) optical transitions within the lowest size-quantized subband, respectively. This conclusion is supported by the calculation of the band structure using an envelope function approximation [17] based on an eight-band $\boldsymbol{k} \cdot \boldsymbol{p}$ Hamiltonian. $\mathrm{HgTe}$, which is a semimetal, forms a type-III QW with $\mathrm{Hg}_{0.3} \mathrm{Cd}_{0.7} \mathrm{Te}$ barrier as indicated in the insets in figure 1. Depending on the quantum confinement, i.e. the well width, and on the temperature, a normal (figure 1(a)) or inverted (figure 1(b)) band structure can be realized with a positive or negative gap between $E_{1^{-}}$and $H_{1}$ subbands [18]. A detailed description of the model as well as the band structure parameters employed in the calculations can be found in [19]. Our calculations show that mid-infrared radiation with a photon energy of the order of $100 \mathrm{meV}$ used in the experiments in fact should cause direct interband optical transitions in all our samples (see figure 1). We note that in some samples transitions between size-quantized subbands may contribute to the absorption of MIR radiation. In the $\mathrm{THz}$ range photon energies are of the order of $\leqslant 10 \mathrm{meV}$, which is insufficient for band-to-band transitions and the current is caused by indirect (Drude-like) optical transitions within the first size-quantized subband.

\section{Experimental results}

Irradiating (0 0 1)-grown $\mathrm{HgTe} / \mathrm{HgCdTe} \mathrm{QW}$ structures with polarized light at oblique incidence we detected a photocurrent signal measured across one of the contact pairs. Figure 2 (left panel) shows the dependence of the photocurrent on the angle $\varphi$ measured at room temperature in the direction normal to the plane of incidence $(x z)$ and obtained at two angles of incidence $\theta_{0}= \pm 30^{\circ}$. The current reversed its direction when switching the sign of the radiation helicity. For the data shown the polarization dependence of the photocurrent 

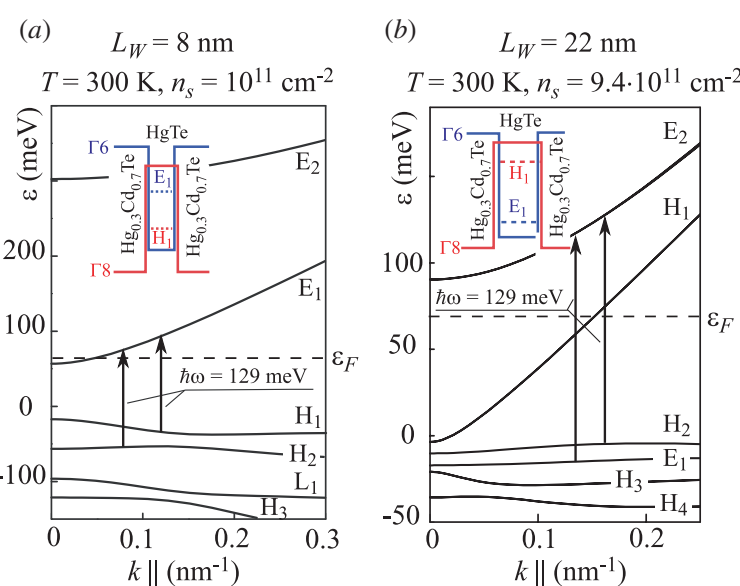

Figure 1. Calculated band structure for QWs with $L_{W}=8 \mathrm{~nm}$ and $22 \mathrm{~nm}$ at $T=300 \mathrm{~K}$. Arrows show optical transitions induced by mid-infrared radiation used in the experiments $(\hbar \omega=129 \mathrm{meV})$. Insets sketch the band profile of HgTe-based QWs with the normal and inverted band structure. Note that while at $T=4.2 \mathrm{~K} 8 \mathrm{~nm}$ QWs have an inverted band structure, at room temperature they exhibit a normal band ordering.

is well described by $J_{y}=J_{0} \cdot \sin 2 \varphi \propto P_{\text {circ }}{ }^{8}$ We emphasize that by inverting the incidence angle the photocurrent changes its sign (at $\theta_{0} \approx 0$ ). In the longitudinal geometry, when the photocurrent is picked up in the plane of incidence, no helicitydependent photocurrent was observed. Below, in section 4, we will demonstrate that these polarization and angle of incidence dependences follow exactly from a phenomenological theory. The effect is observed in wide spectral and temperature ranges, from $6 \mu \mathrm{m}$ to $496 \mu \mathrm{m}$ and from $4.2 \mathrm{~K}$ to $300 \mathrm{~K}$.

Figure 3 shows the wavelength dependence of the circular (photon-helicity-dependent) photocurrent, $J_{\text {circ }}$, obtained for two (0 01 1)-grown QWs. To extract $J_{\text {circ }}$ from the total signal we used the fact that it changes direction upon switching the helicity. Taking the difference of right- and left-handed radiation-induced photocurrents we define $J_{\text {circ }}=\left[J\left(\varphi=45^{\circ}\right)\right.$ $\left.-J\left(\varphi=135^{\circ}\right)\right] / 2$. Figure 3 demonstrates that in the midinfrared range the samples exhibit similar spectral behaviour: the signal does not depend on the wavelength in the range from 6 to $12 \mu \mathrm{m}$ and then rises rapidly, so that from 12 to $15 \mu \mathrm{m}$ the photocurrent strength increases by a factor of 4. While the initial lack of the spectral dependence of $J_{\text {circ }}$ can be attributed to the spectral behaviour of the interband absorption in this range, the signal rise at longer wavelength remains unclear. One of the possible explanations might be attributed to intersubband resonance absorption. However, the wavelength of resonant transitions between the two lowest subbands should depend strongly on the QW width [20], whereas figure 3 shows that the signal increase occurs at the same wavelengths for samples with very different QW widths. This observation excludes resonant intersubband transitions as an origin of such spectral behaviour, unless there is an accidental coincidence of the absorption between the lowest subbands in the narrow well and the absorption into higher

8 We note that at some wavelength of the radiation an additional photocurrent component proportional to $\sin 4 \varphi$ is detected. This contribution is due to the LPGE [16, 20, 21].

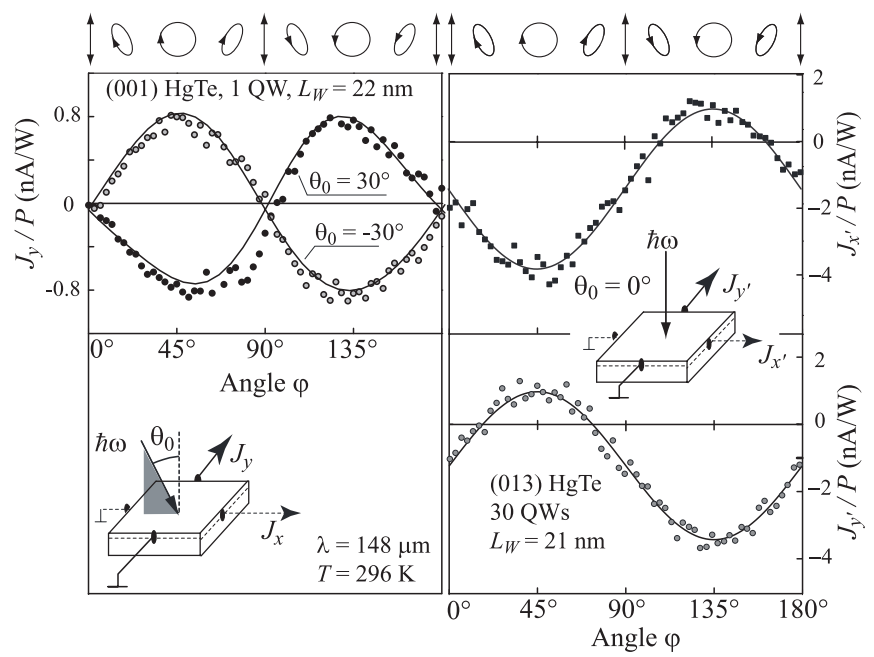

Figure 2. Photocurrent as a function of radiation helicity measured at room temperature in HgTe QWs grown on (0 001$)$ - and (0 13 )-oriented substrates (left and right panels, respectively). The photocurrent was excited by $\mathrm{THz}$ radiation with wavelength $\lambda=148 \mu \mathrm{m}$ and power $P \approx 5 \mathrm{~kW}$. The left panel shows a photocurrent generated in the $\left(\begin{array}{llll}0 & 0 & 1\end{array}\right)$-grown $\mathrm{QW}$ at oblique incidence in the direction normal to the plane of incidence (data are shown for $\theta_{0}= \pm 30^{\circ}$ ). The solid lines are fits to the phenomenological equation (4), where the term proportional to $\sin 2 \varphi$ dominates. The right panels represent the photocurrent in (0 13 )-grown QWs detected at normal incidence $\left(\theta_{0}=0\right)$ in two perpendicular directions. The solid lines are fits to the phenomenological equations (5) and (6). Insets show corresponding experimental geometries. Along the top the polarization ellipses corresponding to key phase angles $\varphi$ are sketched.

subbands in the wider well. This is possible in principle in HgTe-based QWs which have a complex band structure. One could also imagine that, in spite of the fact that direct optical transitions dominate in the absorption of mid-infrared radiation, the weaker free carrier absorption may contribute substantially to the photocurrent generation yielding larger signals at lower frequencies. This assumption, however, is not supported by the measurements carried out in the $\mathrm{THz}$ range (see the inset in figure 3), where current is excited by radiation with photon energy smaller than the energy gap. Figure 3 demonstrates that in the whole $\mathrm{THz}$ range the photocurrent magnitude is substantially smaller than that obtained with mid-infrared radiation. This fact indicates that the photocurrent in the mid-infrared range cannot be attributed to free carrier absorption, because this mechanism should yield an enhancement of the current with the wavelength increase. Such a spectral dependence is indeed observed in the terahertz range, where photocurrent rises significantly at longer wavelengths (see the inset in figure 3 ).

The helicity-dependent photocurrent was also detected in (0 13)-grown samples (see figures 2 and 4). However, in contrast to $\left(\begin{array}{lll}0 & 0 & 1\end{array}\right)$-oriented QWs, in these structures the current is observed even at normal incidence. Here depending on the structure design, radiation wavelength and/or temperature the currents in both $x^{\prime}$ and $y^{\prime}$ directions can be well fitted simply by $J=J_{0} \cdot \sin 2 \varphi \propto P_{\text {circ }}$ (as for the circumstances of 


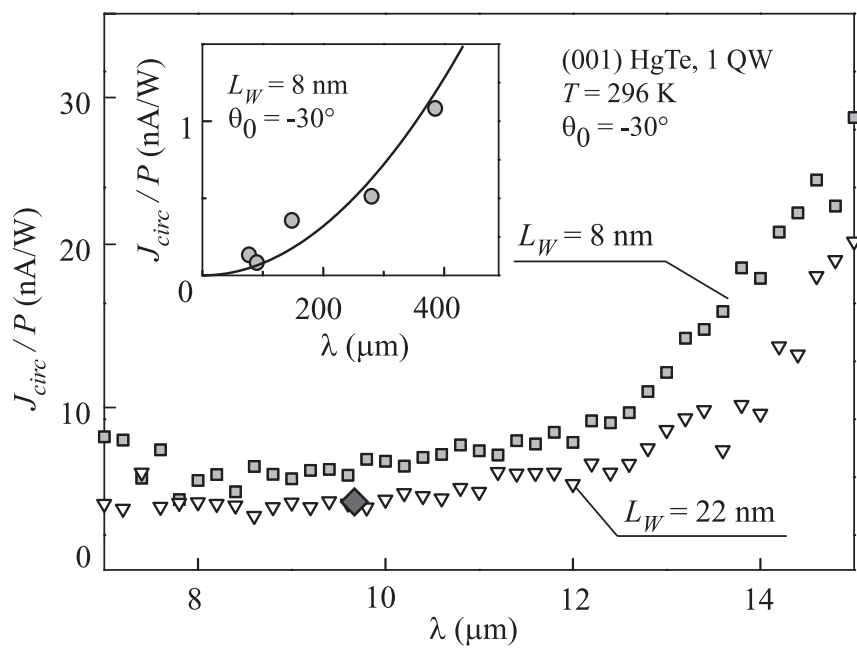

Figure 3. The spectral dependence of the photon-helicity-driven photocurrent measured in two $(001)$-grown structures with QW widths of $L_{W}=22 \mathrm{~nm}$ (triangles) and $L_{W}=8 \mathrm{~nm}$ (squares) in the mid-infrared range. The data were taken using a free electron laser and were scaled to the CPGE photocurrent obtained for $L_{W}=$ $22 \mathrm{~nm}$ found by using a TEA $\mathrm{CO}_{2}$ laser operating at $\lambda=9.6 \mu \mathrm{m}$ (diamond). The inset shows the CPGE photocurrent spectrum in the THz range for $L_{W}=8 \mathrm{~nm}$. The full line is a guide for the eyes.

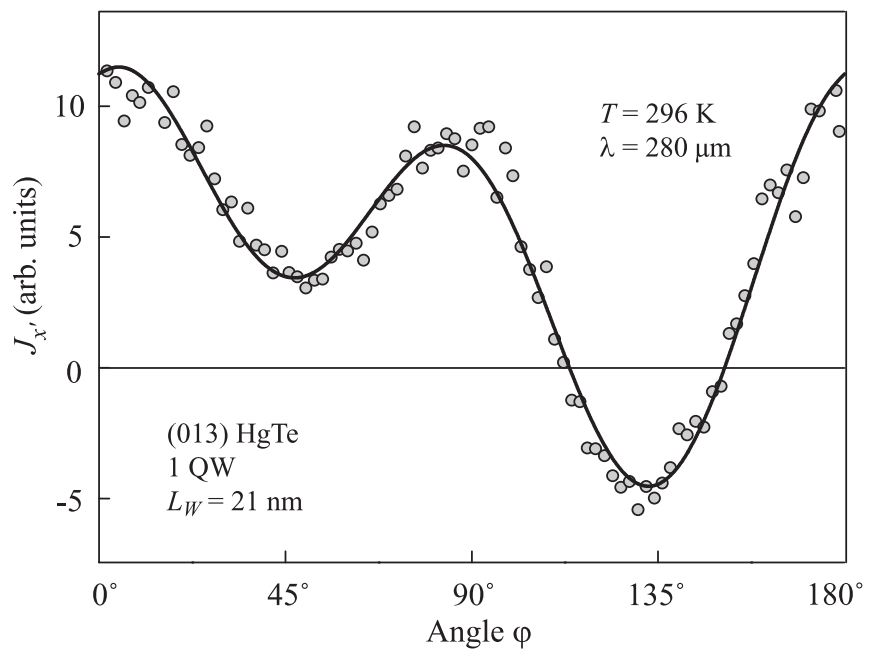

Figure 4. The helicity dependence of the photocurrent in the (0 1 3)-grown HgTe single QW for normal incidence light. The solid line is a fit to the phenomenological equation (6).

figure 2) or by more complex dependence on the angle $\varphi$ (see figure 4) given by

$$
J=A \cdot \sin 2 \varphi+B \cdot \sin 4 \varphi+C \cdot \cos 4 \varphi+D .
$$

Here, $A, B, C$ and $D$ are the fitting parameters, described in more detail below in section 4 . While the first term on the right-hand side of (1) describes the CPGE [6], the other terms yield the LPGE $[16,20,21]$. We note that even if $B, C$ and $D$ are non-zero, $J_{\text {circ }}=\left[J\left(\varphi=45^{\circ}\right)-J\left(\varphi=135^{\circ}\right)\right] / 2$ is proportional to $A$ and hence is a measure of the CPGE. Measuring the CPGE and LPGE contributions as a function of the angle of incidence we observed that all currents for the (0 1 3)-grown samples reach a maximal value at normal incidence. Below we focus on the CPGE.

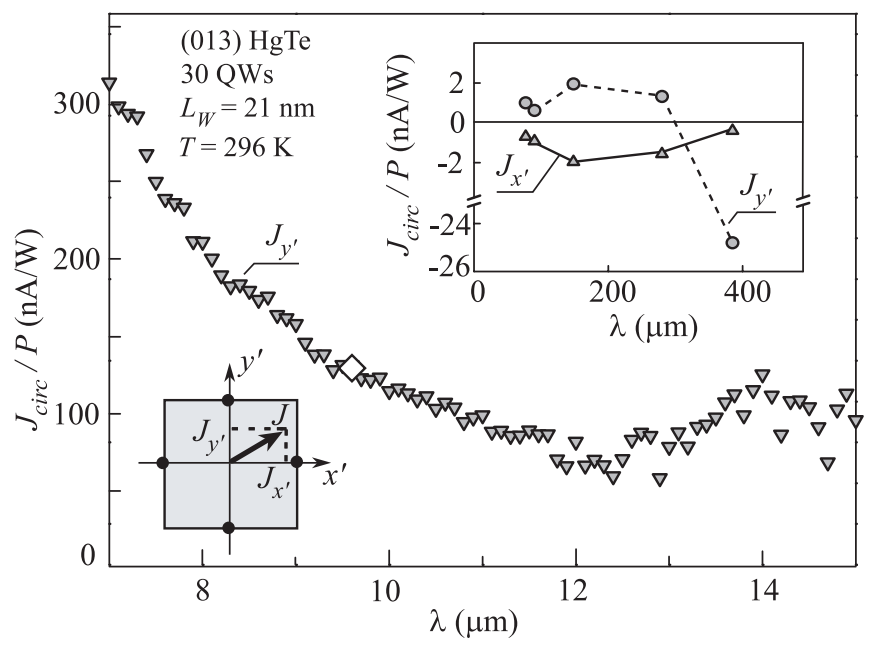

Figure 5. The spectral behaviour of the power-normalized helicity-dependent photocurrent along the $x^{\prime}$ direction measured in the (0 1 3)-grown QW excited by the mid-infrared radiation. The data are obtained at normal incidence using the free electron laser (down triangles) and normalized to the CPGE photocurrent measured by applying the TEA $\mathrm{CO}_{2}$ laser (diamond) operating at $\lambda=9.6 \mu \mathrm{m}$. The top right inset shows spectral behaviour of $J_{x^{\prime}}$ and $J_{y^{\prime}}$ in the THz range, which correspond to the projections of the total photocurrent (see the inset in the bottom, left). Note the axis break on the ordinate.

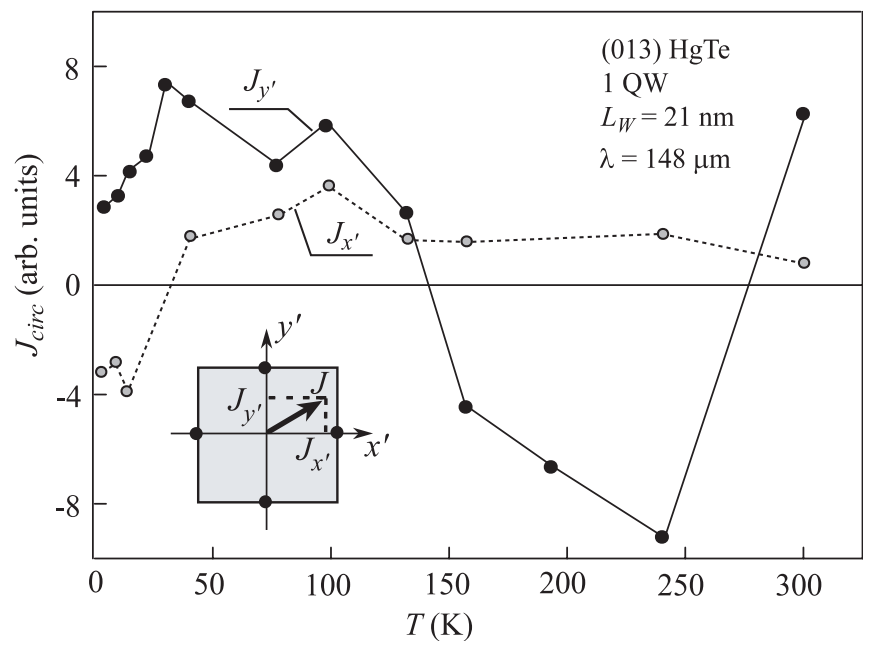

Figure 6. Temperature dependence of $J_{x^{\prime}}$ and $J_{y^{\prime}}$ photocurrents measured in the (0 1 13$)$-grown single QW applying radiation with $\lambda=148 \mu \mathrm{m}$. The photocurrents correspond to the projections of the total photocurrent on the $x^{\prime}$ and $y^{\prime}$ directions (see the inset).

Experimentally, we observed a major difference between the photocurrent excited in (0 1 3)-oriented QWs and that in ( $\left.\begin{array}{lll}0 & 0 & 1\end{array}\right)$-grown QWs. In the higher symmetry structures, $\left(\begin{array}{lll}0 & 0 & 1\end{array}\right)$ grown QWs, $J_{\text {circ }}$ can only be excited by oblique incidence, its direction is always restricted to the perpendicular to the plane of incidence for the experimental geometry used, and only the photocurrent magnitude and sign are the functions of angle, temperature and wavelength. In the (0 1 13$)$-grown structure, $J_{\text {circ }}$ can be produced by normal incidence and can flow in any direction as shown in figures 2,5 and 6 , where it can be seen that the photocurrent components $x^{\prime}$ and $y^{\prime}$ behave differently and even change their relative sign depending on 
the wavelength and temperature. The spectral behaviour of the circular photocurrent is shown in figure 5 for one of the pairs of contacts. As for $\left(\begin{array}{ll}0 & 0\end{array}\right)$-grown QWs, we observed that for some mid-infrared spectral range (in this case 10-15 $\mu \mathrm{m}$ ) the signal is almost independent of the wavelength. Unlike (0 01 l)-grown QWs, it becomes larger at shorter $\lambda$. Applying THz radiation we observed the photocurrent caused by free carrier absorption which, like for (001)-grown QWs, is substantially smaller than that in mid-infrared range. The wavelength and the temperature dependence of $J_{x^{\prime}}$ and $J_{y^{\prime}}$ components are shown in the insets in figures 5 and 6 , respectively. We emphasize that the lack of restriction on the photocurrent direction was detected for (0 1 3)-grown QWs only, which are characterized by the point symmetry group $\mathrm{C}_{1}$, the group of the lowest symmetry comprising only the identity.

\section{Phenomenological theory and discussion}

In order to describe the observed dependences of photocurrent on the light polarization and the angle of incidence, as well as to understand the qualitative difference in photocurrents in (0 01 )- and (0 13)-grown structures, we derive here phenomenological equations for the linear and circular PGE in two-dimensional HgTe-based structures. The photogalvanic current density $\boldsymbol{j}$ can be written as a function of the electric component $\boldsymbol{E}$ of the radiation field and the propagation direction $\hat{e}$ in the following form $[5,20]$ :

$$
j_{\lambda}=\sum_{\beta} \gamma_{\lambda \beta} \hat{e}_{\beta} P_{\text {circ }}|E|^{2}+\sum_{\mu, \nu} \chi_{\lambda \mu \nu} \frac{E_{\mu} E_{\nu}^{*}+E_{\mu}^{*} E_{\nu}}{2},
$$

where the first term on the right-hand side is proportional to the radiation helicity $P_{\text {circ }}$ and represents the CPGE, while the second term corresponds to the linear photogalvanic effect $[16,20]$, which may be superimposed on the CPGE. The indices $\lambda, \beta, \mu, v$ run over the coordinate axes. The second rank pseudotensor $\gamma$ and the third rank tensor $\chi$, symmetric in the last two indices, are the material parameters. We note that while in the theoretical consideration the current density $\boldsymbol{j}$ is used, in the experiments the electric current $\boldsymbol{J}$ is measured which is proportional to $j$.

The HgTe QWs grown along the $z \|$ [0 01 1] direction correspond to the $\mathrm{C}_{2 \mathrm{v}}$ point group. This group includes a rotating axis $\mathrm{C}_{2}$ parallel to the [0 011$]$-direction and two mirror planes, $m_{1}$ and $m_{2}$, coinciding with the $(x z)$ and $(y z)$ planes, respectively (figure 7). It follows from Neumann's principle and equation (2) that the circular photocurrent can only occur along those axes where for all symmetry operations components of $\boldsymbol{j}$ transform in the same way as components of the pseudovector $\boldsymbol{S}=P_{\text {circ }} \hat{\boldsymbol{e}}$ describing the radiation helicity. Let us consider it for circularly polarized radiation propagating along the $x$ direction, i.e. for $S_{x}$. The reflection in each mirror plane transforms the current component $j_{y}$ and the pseudovector component $S_{x}$ in the same way: $j_{y} \rightarrow-j_{y}$, $S_{x} \rightarrow-S_{x}$ for the plane $m_{1}$ (see figure $7(c)$ ) and $j_{y} \rightarrow j_{y}$, $S_{x} \rightarrow S_{x}$ for the plane $m_{2}$. Therefore, the photocurrent $j_{y} \propto S_{x}$ is possible. Similar arguments hold for $j_{x} \propto S_{y}$. For any other relative directions of $\boldsymbol{j}$ and $\boldsymbol{S}$, a linear coupling of the current and the radiation helicity is forbidden. For instance, (a)

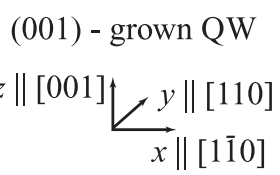

(b)

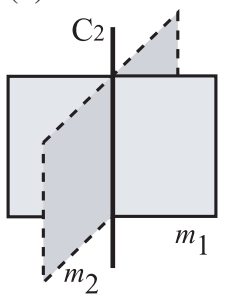

(c) top view

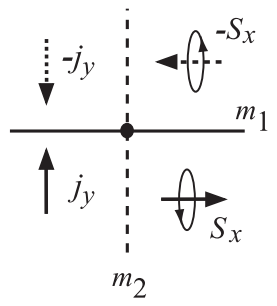

Figure 7. (a) Coordinate system of the (0 0 1)-grown HgTe QW which has $\mathrm{C}_{2 \mathrm{v}}$ symmetry, $(b)$ mirror planes $m_{1}$ and $m_{2}$ and the $\mathrm{C}_{2}$-axis in the QW grown along $z \|\left[\begin{array}{lll}0 & 0 & 1\end{array}\right]$. Arrows in $(c)$ show that the reflection in the mirror plane $m_{1}$ changes the sign of both the polar vector component $j_{y}$ and the axial vector component $S_{x}=P_{\text {circ }} \hat{e}_{x}$, demonstrating that a linear coupling $j_{y} \propto S_{x}$ is allowed under these symmetry operations. This coupling is also allowed by the other symmetry operations of the point group, and so is $j_{x} \propto S_{y}$.

the reflections in both $m_{1}$ and $m_{2}$ planes reverse the direction of $S_{z}$ while $\mathrm{C}_{2}$ does nothing. There is no polar vector component that transforms in the same way. It indicates that $S_{z}$ cannot give rise to a photocurrent in $\left(\begin{array}{lll}0 & 0 & 1\end{array}\right)$-grown $\mathrm{QW}$ structures. Thus, the CPGE can only be generated at oblique incidence and in the direction normal to the plane of incidence, $(x z)$ or $(y z)$, as observed in the experiments. For the QW structures of the $\mathrm{C}_{2 \mathrm{v}}$ point group, these arguments can be used to show that the non-zero components of the second rank pseudotensor and the third rank tensor are the following: $\gamma_{x y}, \gamma_{y x}, \chi_{x x z}=\chi_{x z x}$ and $\chi_{y y z}=\chi_{y z y}$. These four components are linearly independent. Optical excitation of such structures at oblique incidence in the $(x z)$-plane with elliptically polarized light generates electric current whose $x$ - and $y$-components depend on the angles $\varphi$ and $\theta$ as follows:

$$
\begin{aligned}
& j_{x}(\varphi)=\frac{1}{2} \chi_{x x z} E_{0}^{2} t_{p}^{2} \sin \theta \cos \theta(1-\cos 4 \varphi), \\
& j_{y}(\varphi)=E_{0}^{2} t_{p} t_{s} \sin \theta\left[\gamma_{y x} \sin 2 \varphi-\frac{1}{2} \chi_{y y z} \sin 4 \varphi\right] .
\end{aligned}
$$

Here, $E_{0}$ is the electric field amplitude of the incident radiation, $t_{p}$ and $t_{s}$ are the Fresnel amplitude transmission coefficients from vacuum to the structure for the $s$ - and $p$-polarized light, respectively [22], $\theta$ is the refraction angle related to the incidence angle $\theta_{0}$ by $\sin \theta=\sin \theta_{0} / n_{\omega}$ and $n_{\omega}$ is the refractive index.

We note that the LPGE described by equation (3) and second term on the right-hand side of equation (4) was detected for several wavelengths. It can be excited by linearly polarized radiation, which was also checked experimentally (the data are not shown here).

Now we turn to the symmetry analysis of equation (2) for (0 13)-oriented QWs, which was not carried out so far. The QWs of this orientation belong to the trivial point group $\mathrm{C}_{1}$ lacking any symmetry operation except the identity. Hence, symmetry does not impose any restriction on the relation between the radiation electric field and photocurrent components. All components of the pseudotensor $\gamma$ and the tensor $\chi$ may be different from zero. Phenomenologically, for the $C_{1}$ symmetry group, the photogalvanic current for the 
excitation along the QW normal with elliptically polarized light is given by

$$
\begin{aligned}
j_{x^{\prime}}= & -E_{0}^{2} t_{s}^{2}\left[\gamma_{x^{\prime} z^{\prime}} \sin 2 \varphi-\frac{\chi_{x^{\prime} x^{\prime} x^{\prime}}+\chi_{x^{\prime} y^{\prime} y^{\prime}}}{2}\right. \\
& \left.+\frac{\chi_{x^{\prime} x^{\prime} x^{\prime}}-\chi_{x^{\prime} y^{\prime} y^{\prime}}}{4}(1+\cos 4 \varphi)+\frac{\chi_{x^{\prime} x^{\prime} y^{\prime}}}{2} \sin 4 \varphi\right],
\end{aligned}
$$

$$
\begin{aligned}
j_{y^{\prime}}= & -E_{0}^{2} t_{s}^{2}\left[\gamma_{y^{\prime} z^{\prime}} \sin 2 \varphi-\frac{\chi_{y^{\prime} x^{\prime} x^{\prime}}+\chi_{y^{\prime} y^{\prime} y^{\prime}}}{2}\right. \\
& \left.+\frac{\chi_{y^{\prime} x^{\prime} x^{\prime}}-\chi_{y^{\prime} y^{\prime} y^{\prime}}}{4}(1+\cos 4 \varphi)+\frac{\chi_{y^{\prime} x^{\prime} y^{\prime}}}{2} \sin 4 \varphi\right] .
\end{aligned}
$$

Exactly this polarization dependence is encapsulated in (1) and observed in experiments as can be seen in figure 2 and, in particular, in figure 4, where all terms given by (6) contribute substantially. Equation (2) shows that the photogalvanic current may also be excited by linearly polarized light in the geometry of normal incidence. In this case, equations (5) and (6) take the form

$$
\begin{aligned}
j_{x^{\prime}}= & -E_{0}^{2} t_{s}^{2}\left[\chi_{x^{\prime} x^{\prime} y^{\prime}} \sin 2 \alpha-\frac{\chi_{x^{\prime} x^{\prime} x^{\prime}}+\chi_{x^{\prime} y^{\prime} y^{\prime}}}{2}\right. \\
& \left.+\frac{\chi_{x^{\prime} x^{\prime} x^{\prime}}-\chi_{x^{\prime} y^{\prime} y^{\prime}}}{2} \cos 2 \alpha\right]
\end{aligned}
$$

$$
\begin{aligned}
j_{y^{\prime}}= & -E_{0}^{2} t_{s}^{2}\left[\chi_{y^{\prime} x^{\prime} y^{\prime}} \sin 2 \alpha-\frac{\chi_{y^{\prime} x^{\prime} x^{\prime}}+\chi_{y^{\prime} y^{\prime} y^{\prime}}}{2}\right. \\
& \left.+\frac{\chi_{y^{\prime} x^{\prime} x^{\prime}}-\chi_{y^{\prime} y^{\prime} y^{\prime}}}{2} \cos 2 \alpha\right]
\end{aligned}
$$

where $\alpha$ is the angle between the light polarization plane and the $x^{\prime}$ axis. We have checked experimentally (not shown) that the photocurrent induced under linearly polarized excitation is well fitted by these equations.

Equations (5) and (6) show that, in (0 13 )-oriented QWs, the CPGE photocurrent direction is arbitrary and not forced to a definite crystallographic axis. The fact that $J_{x^{\prime}}$ and $J_{y^{\prime}}$ as well as their ratio $J_{x^{\prime}} / J_{y^{\prime}}$ exhibit a nontrivial variation with the radiation wavelength (figure 5) or sample temperature (figure 6) is also in agreement with equations (5) and (6) yielding for circularly polarized light $j_{x^{\prime}} / j_{y^{\prime}}=\gamma_{x^{\prime} z^{\prime}} / \gamma_{y^{\prime} z^{\prime}}$. Indeed all the components of the tensor $\gamma$ are linearly independent in the structures of the $\mathrm{C}_{1}$ point-group symmetry, so the ratio between them can be changed by varying the experimental conditions. Therefore there is no preferential direction of the circular photocurrent forced by the symmetry arguments. The same is valid, and observed experimentally, for the linear photogalvanic current.

Another important point is that the photocurrent strength in HgTe-based structures, e.g. for the CPGE current, is of about an order of magnitude larger than that observed in GaAs, InAs and SiGe low-dimensional structures [5]. Our results reveal that photogalvanic measurements open a rich field for the investigation of the microscopic properties of this novel and promising material. The microscopic theory of these phenomena in HgTe-based QWs, in particular with the inverted band ordering, is a task for future, which is complicated by the band structure, with almost flat valence bands and strong spin slitting in comparison with other materials. In fact, all known mechanisms of the CPGE are enhanced in HgTe-based structures: spin-related mechanisms [5, 6, 8, 23] due to large spin-orbit coupling, and orbital mechanisms [9, 10] due to a very small band gap. To distinguish between spin and orbital mechanisms additional research is required. In this respect future experiments on time-resolved photogalvanics under short-pulsed circularly polarized photoexcitation, with the pulse duration being comparable with the free-carrier momentum and spin relaxation times, would be desirable and informative. Such experiments would also reveal a great deal about the momentum, energy and spin relaxation of nonequilibrium photoexcited carriers.

\section{Conclusions}

To summarize, our experiments show that helicity-driven photogalvanic currents can effectively be generated in $\mathrm{HgTe}$ QWs. The large photoresponse obtained in the whole range of investigated radiation frequencies suggests that $\mathrm{HgTe}$ QW structures are promising for the detection of $\mathrm{THz} / \mathrm{IR}$ radiation, in particularly, for the all-electric detection of the radiation Stokes parameters [24]. Our results, however, reveal that, while (0 1 3)-grown QWs can be effectively used for certain radiation wavelengths, they cannot be applied for a broadband detection. As discussed above, in structures of this orientation, symmetry does not impose any restriction on the relation between the radiation electric field and photocurrent components resulting in a complex spectral and temperature behaviour. To get around this complexity (which would make calibration difficult) but preserve the possibility of use at normal incidence, (1 12 )-grown QWs could be used. Such QWs are technologically available, and belong to the $\mathrm{C}_{s}$ point group, confining the CPGE current to the [1 10 l 0 crystallographic direction for normal incidence radiation. This feature makes it possible to prepare devices for which the CPGE dominates at all wavelengths.

\section{Acknowledgments}

We thank E L Ivchenko for helpful discussions. The financial support of the DFG via programs SFB 689 and AS327/2-1, the Linkage grant of IB of BMBF at DLR, the MES, the RFBR, the President Grant for young scientists (MD-1717.2009.2), and Foundation 'Dynasty'-ICFPM is gratefully acknowledged.

\section{References}

[1] Buhmann H 2009 Int. J. Mod. Phys. B 232551

[2] König M et al 2007 Science 318766

[3] Gui Y S et al 2004 Phys. Rev. B 70115328

[4] Diehl H et al 2009 Phys. Rev. B 80075311

[5] Ivchenko E L and Ganichev S D 2008 Spin photogalvanics Spin Physics in Semiconductors ed M I Dyakonov (Berlin: Springer)

[6] Ganichev S D et al 2001 Phys. Rev. Lett. 864358

[7] Ganichev S D and Prettl W 2003 J. Phys.: Condens. Matter 15 R935

[8] Bieler M, Laman N, van Driel H M and Smirl A L 2005 Appl. Phys. Lett. 86061102 
[9] Tarasenko S A 2007 JETP Lett. 85182

[10] Olbrich P et al 2009 Phys. Rev. B 79121302

[11] Deyo E, Golub L E, Ivchenko E L and Spivak B 2009 Semiclassical theory of the photogalvanic effect in non-centrosymmetric systems arXiv:0904.1917

[12] Moore J E and Orenstein J 2009 Confinement-induced Berry phase and helicity-dependent photocurrents arXiv:0911.3630

[13] Ganichev S D et al 2008 J. Appl. Phys. 103114504

[14] Varavin V S et al 2003 Proc. SPIE 3815136

[15] Knippels G M H et al 1999 Phys. Rev. Lett. 831578

[16] Ganichev S D and Prettl W 2006 Intense Terahertz Excitation of Semiconductors (Oxford: Oxford University Press)
[17] Burt M G 1992 J. Phys.: Condens. Matter 46651 Burt M G 1999 J. Phys.: Condens. Matter 11 R53 Foreman B A 1993 Phys. Rev. B 484964

[18] König M et al 2008 J. Phys. Soc. Japan 77031007

[19] Novik E G et al 2005 Phys. Rev. B 72035321

[20] Ivchenko E L 2005 Optical Spectroscopy of Semiconductor Nanostructures (Harrow, UK: Alpha Science Int.)

[21] Sturman B I and Fridkin V M 1992 The Photovoltaic and Photorefractive Effects in Non-Centrosymmetric Materials (NY: Gordon and Breach)

[22] Born M and Wolf E 1970 Principles of Optics (Oxford: Pergamon)

[23] Ganichev S D et al 2003 Phys. Rev. B 68035319

[24] Danilov S N et al 2009 J. Appl. Phys. 105013106 